Pacific Journal of Mathematics

SOME GENERALIZATIONS OF METRIC SPACES 


\title{
SOME GENERALIZATIONS OF METRIC SPACES
}

\author{
JACK G. CEDER
}

1. Introduction. This paper consists of a study of certain classes of topological spaces (called $M_{1}-, M_{2}$, and $M_{3}$-spaces) which include metric spaces and $C W$-complexes and are included in the class of all paracompact and perfectly normal spaces. It is shown, for example, that like the case in metric spaces, a subset of an $M_{2^{-}}$(or $M_{3^{-}}$) space is an $M_{2^{-}}$(or $M_{3^{-}}$) space; a countable product of $M_{i}$-spaces $(i=1,2,3)$ is again an $M_{i}$-space; and separable is equivalent to Lindelöf in an $M_{i}$-space. Moreover, unlike the case in metric spaces, the quotient space obtained by identifying the points of a closed subset of an $M_{2^{-}}$(or $M_{3^{-}}$) space is again an $M_{2^{-}}$(or $M_{3^{-}}$) space (for metric spaces such a quotient space need not be first countable). Also, we have $M_{1} \rightarrow M_{2} \rightarrow M_{3}$, but whether $M_{3} \rightarrow M_{2}$ or $M_{2} \rightarrow M_{1}$ is unknown. ${ }^{1}$

These classes of spaces are derived from generalizations of the following well-known characterization of metrizability in terms of specific properties of the base:

Theorem 1.1. (Smirnov [14] or Nagata [12]). A regular space is metrizable if and only if it has a $\sigma$-locally finite base.

Recall that a $\sigma$-locally finite family is a union of countably many locally finite families. It is easily checked that a locally finite family $\boldsymbol{U}$ of sets has the property, called closure preserving, that for any

$$
\boldsymbol{V} \subset \boldsymbol{U}, \quad(\cup\{V \in \boldsymbol{V}\})^{-}=\cup\{V: V \in \boldsymbol{V}\} .
$$

This, then, suggests we consider spaces having a $\sigma$-closure preserving base (that is, a base which is the union of countably many closure preserving families).

Definition 1.1. An $M_{1}$-space is a regular space having a $\sigma$-closure preserving base.

Although conceptually simple, $M_{1}$-spaces prove unsatisfactory in some respects, so we weaken the condition of having a $\sigma$-closure preserving base. We begin by calling a collection $\boldsymbol{B}$ of (not necessarily open!) subsets of $X$ a quasi-base if, whenever $x \in X$ and $U$ is a neighborhood of

Received June 12, 1959, resubmitted November 13, 1959. This paper represents part of the authors doctoral dissertation at the University of Washington, prepared under the guidance of Professor E. A. Michael, to whom the author wishes to express his gratitude for his advice and encouragement. The author is also indebted to Professor Jun-iti Nagata for some helpful correspondence.

1 Nearly all topological terminology appearing in this paper is consistent with that used in Kelley [4]. Exceptions are that our regular, and normal spaces are assumed to be $T_{1^{-}}$. spaces. 
$x$, then there exists a $B \in B$ such that $x \in B^{0} \subset B \subset U$ where $B^{0}$ denotes the interior of $B$ ).

DefinItion 1.2. An $M_{\varepsilon}$-space is a regular space with a $\sigma$-closure preserving quasi-base.

Now we proceed to weaken the condition of having a $\sigma$-closure preserving quasi-base. Let $\boldsymbol{P}$ be a collection of ordered pairs $P=\left(P_{1}, P_{2}\right)$ of subsets of $X$, with $P_{1} \subset P_{2}$ for all $P \in P$. Then $\boldsymbol{P}$ is called a pairbase for $X$ if $P_{1}$ is open for all $P \in P$ and if, for any $x \in X$ and neighborhood $U$ of $x$, there exists a $P \in P$ such that $x \in P_{1} \subset P_{2} \subset U$. Moreovor, $\boldsymbol{P}$ is called cushioned if for every $\boldsymbol{P}^{\prime} \subset \boldsymbol{P}$,

$$
\left(\bigcup\left\{P_{1}: P \in P^{\prime}\right\}\right)^{-} \subset \bigcup\left\{P_{2}: P \in P^{\prime}\right\} .
$$

$\boldsymbol{P}$ is called $\sigma$-cushioned if it is the union of countably many cushioned subcollections.

Definition 1.3. An $M_{3}$-space is a $T_{1}$-space with a $\sigma$-cushioned rairbase.

\section{Properties of $M_{i}$-spaces.}

TheOREM 2.1. (Michael [6]). A $T_{1}$-space is paracompact if and only if every open cover $\boldsymbol{U}$ has a $\sigma$-cushioned open refinement $\boldsymbol{V}$ (that is, $\boldsymbol{V}=\bigcup_{n=1}^{\infty} \boldsymbol{V}_{n}$, where for each $n$, and $V \in \boldsymbol{V}_{n}$ one can assign a $U_{V, n} \in \boldsymbol{U}$ such that $\left\{\left(V, U_{V . n}\right): V \in \boldsymbol{V}_{n}\right\}$ is cushioned).

THEOREM 2.2. The following implications hold: Metrizable $\rightarrow M_{1} \rightarrow$ $M_{2} \rightarrow M_{3} \rightarrow$ paracomxact and perfectly normal.

Proof. Metrizable $\rightarrow M_{1}$ and $M_{1} \rightarrow M_{2}$ are obvious.

To show $M_{2} \rightarrow M_{3}$, let $\bigcup_{n=1}^{\infty} \boldsymbol{B}_{n}$ be a $\sigma$-closure preserving quasi-base. For each $n$, put $\boldsymbol{P}_{n}=\left\{\left(B^{0}, \bar{B}\right): B \in \boldsymbol{B}_{n}\right\}$. Then clearly $\bigcup_{n=1}^{\infty} \boldsymbol{P}_{n}$ becomes a $\sigma$-cushioned pair-base.

To show $M_{3} \rightarrow$ paracompactness, let $\bigcup_{n=1}^{\infty} \boldsymbol{P}_{n}$ be a $\sigma$-cushioned pairbase. Let $\boldsymbol{U}$ be an open cover and for each $n$, let $W=\left\{P_{1} \subset P_{2} \subset U_{W, n}\right.$ for some $\left.U \in \boldsymbol{U}, U \in \boldsymbol{P}_{n}\right\}$. For $W \in \boldsymbol{W}_{n}$, pick $U_{W, n} \in \boldsymbol{U}$ such that for some $P \in \boldsymbol{P}_{n}, W=P_{1} \subset P_{2} U_{W, n}$. Then $\boldsymbol{W}=\bigcup_{n=1}^{\infty} \boldsymbol{W}_{n}$ becomes a $\sigma$-cushioned open refinement of $U$ and hence, by Theorem 2.1, $X$ is paracompact.

To show $M_{3} \rightarrow$ perfectly normal, let $G$ be an open set in $X$. For each $n$, put $F_{n}=\left(\bigcup\left\{P_{1}: P_{2} \subset G, P \in P_{n}\right\}\right)^{-}$. Then $G=\bigcup_{n=1}^{\infty} F_{n}$, so every open set is an $F_{\sigma}$, whence $X$ is perfectly normal since $X$ is normal by paracompactness, thus completing the proof of Theorem 2.2.

Example 9.2 furnishes us with a separable and first countable $M_{1}$ space which is non-metrizable. The "half-open interval" space $R$ (the 
real line $R$ with base the family $\{[x, y): x, y \in R\}$ is paracompact and perfectly normal and $R \times R$ is not paracompact (Sorgenfrey [16] or Kelley [4]). Hence, by Theorem 2.2, $R \times R$ is not $M_{3}$, and by Theorem 2.4 it follows that $R$ is not $M_{3}$. The questions of whether $M_{2} \rightarrow M_{1}$ or $M_{3} \rightarrow M_{2}$ remain unsolved. However, see Proposition 7.7 for a partial result.

The following three theorems exhibit properties which metric spaces have in common with $M_{i}$-spaces.

Theorem 2.3. If $A$ is a subset of an $M_{2^{-}}$(or $M_{3^{-}}$) space $X$, then $A$ is $M_{2}\left(\right.$ or $\left.M_{3}\right)$.

Proof. We prove it only for the $M_{2}$-case. Let $\bigcup_{n=1}^{\infty} \boldsymbol{B}_{n}$ be a $\sigma$-closure preserving quasi-base for $X$. For each $n$, put $\boldsymbol{B}_{n}^{\prime}=\left\{A \cap \bar{B}: B \in \boldsymbol{B}_{n}\right\}$. To show $\boldsymbol{B}_{n}^{\prime}$ is closure preserving in $A$ it suffices to show for $x \in A$ and $\boldsymbol{A} \subset \boldsymbol{B}_{n}$, that $x \notin \cup\left\{(A \cap \bar{B})^{-}: B \in \boldsymbol{A}\right\}$ implies $x \notin(\cup\{A \cap \bar{B}: B \in \boldsymbol{A}\})^{-}$. But for any $B \in \boldsymbol{A}, x \notin(A \cap \bar{B})^{-}$implies $x \notin A \cap \bar{B}$ and $x \notin \bar{B}$. So $x \notin \cap\{\bar{B}: B \in \boldsymbol{A}\}=\left(\cup\{\bar{B}: B \in \boldsymbol{A})^{-}\right.$and hence, $x \notin(\cup\{A \cap \bar{B}: B \in \boldsymbol{A}\})^{-}$ and $\boldsymbol{B}_{n}^{\prime}$ is closure preserving. Let $U$ be open about $x$ in $A$. Then for some $U^{\prime}$ open in $X$ we have $U=U^{\prime} \cap A$, so there exists $B$ in some $\boldsymbol{B}_{n}$ so that $x \in B^{0} \subset B \subset \bar{B} \subset U^{\prime}$. Then with $A \cap \bar{B} \in \boldsymbol{B}_{n}^{\prime}$, we have $x \equiv\left(B^{0} \cap A\right) \subset$ $(A \cap \bar{B})^{0} \subset(A \cap \bar{B}) \subset\left(U^{\prime} \cap A\right)=U$. Hence $A$ is $M_{2}$, which completes the proof.

The foregoing proof breaks down in the case of an $M_{1}$-space (since in general $\left.\left(B^{0} \cap A\right)^{-} \neq(A \cap \bar{B})\right)$, and it is unsolved whether a subspace, or even a closed subspace, of an $M_{1}$-space is $M_{1}$.

THeORem 2.4. A countable product of $M_{i}$-spaces is $M_{i}$.

Proof. We prove it only for the $M_{1}$ case; the other cases follow similarly. For each $n$, let $X_{n}$ be an $M_{1}$-space with a $\sigma$-closure preserving base $\bigcup_{m=1}^{\infty} \boldsymbol{B}_{n}^{m}$. Without loss of generality we can assume that, for all $m, n, X_{n} \in \boldsymbol{B}_{n}^{m}$ and $\boldsymbol{B}_{n}^{m} \subset \boldsymbol{B}_{n}^{m+1}$. Now put $X=\prod_{n=1}^{\infty} X_{n}$ and, for each $n$, let

$$
\boldsymbol{B}_{n}=\prod_{i=1}^{n}\left\{B_{i}: B_{i} \in \boldsymbol{B}_{i}^{n}\right\}
$$

where

$$
\prod_{i=1}^{n} B_{i}=\left\{x \in X: x_{i} \in B_{i} \text { for } i \leqq n\right\} .
$$

Then $\bigcup_{n=1}^{\infty} \boldsymbol{B}_{n}$ becomes a $\sigma$-closure preserving base for $X$, making $X$ an $M_{1}$-space.

We can also prove the following result: 
Theorem 2.5. Let $X$ be an $M_{i}$-space. Then the following are equivalent:

(1) $X$ is separable,

(2) $X$ is Lindelöf,

(3) $X$ is satisfies the countable chain condition (that is, every disjoint family of open sets is countable).

A separable $M_{1}$-space need not have a countable base; for example, see Example 9.2.

Smirnov [15] has shown that any locally metrizable paracompact space is metrizable. And Nagata [13] has obtained the stronger result that a space which is the union of a locally finite family of closed metrizable subsets in metrizable. We can obtain analogous results as follows:

TheOREM 2.6. If $X$ is paracompact and locally $M_{i}$, then $X$ is $M_{i}$.

Proof. We prove it only for the $M_{1}$ case, and note that the others follow analogously. For each $x \in X$, there exists an open neighborhood $W(x)$ of $x$ such that $W(x)$ is $M_{1}$. By paracompactness, let $\left\{U_{\alpha}: \alpha \in A\right\}$ be an open locally finite refinement of $\{W(x): x \in X\}$. Then, since an open subset of an $M_{1}$-space is clearly $M_{1}$, each $U_{\alpha}$ is $M_{1}$. Let $\boldsymbol{B}^{\alpha}=$ $\bigcup_{n=1}^{\infty} \boldsymbol{B}_{n}^{\alpha}$ be a $\sigma$-closure preserving base for $U_{\alpha}$ such that, for each $B \in \boldsymbol{B}^{\alpha}$, $\bar{B} \subset U_{\alpha}$. For each $n$, put $\boldsymbol{C}_{n}=\bigcup\left\{\boldsymbol{B}_{n}^{\alpha}: \alpha \in A\right\}$. Then it easily follows that each $\boldsymbol{C}_{n}$ is closure preserving and $\bigcup_{n=1}^{\infty} \boldsymbol{C}_{n}$ is a base for $X$.

LEMmA 2.7. If $X=A_{1} \cup A_{2}$, where $A_{1}$ and $A_{2}$ are closed $M_{2^{-}}$(or $M_{3^{-}}$) subspaces, then $X$ is $M_{2}$ (or $\left.M_{3}\right)$.

Proof. First we get $X$ to be regular (Nagata [12]). For the $M_{2}$ case, let $\bigcup_{n=1}^{\infty} B_{n}^{1}$ and $\bigcup_{n=1}^{\infty} B_{n}^{2}$ be $\sigma$-closure preserving quasi-bases for $A_{1}$ and $A_{2}$ respectively, with $\phi \in \boldsymbol{B}_{n}^{1} \cap \boldsymbol{B}_{n}^{2}$ for all $n$. Now for each $n, m$, we put $\boldsymbol{B}_{n, m}=\left\{B_{1} \cup B_{2}: B_{1} \in \boldsymbol{B}_{n}^{1}, B_{2} \in \boldsymbol{B}_{m}^{2}\right\}$. Then it is easily checked that $\bigcup_{n, m=1}^{\infty} \boldsymbol{B}_{n, m}$ is a $\sigma$-closure preserving quasi-base for $X$. Hence $X$ is $M_{2}$. The $M_{3}$ case is similar.

THEOREM 2.8. If $X$ is a locally finite union of closed $M_{2^{-}}$(or $M_{3^{-}}$) spaces, then $X$ is $M_{2}$ (or $M_{3}$ ).

Proof. First we apply a theorem of Michael [7, pp. 379-380] and Morita [10] (see Theorem 8.1 of this paper) to get $X$ paracompact. Let $X$ be the union of a locally finite family $\boldsymbol{A}$ of closed $M_{2^{-}}$( or $M_{3^{-}}$) spaces. Then, for each $x \in X$, there exists an open $U_{x}$ containing $x$ which intersects only finitely many members of $\boldsymbol{A}$, say $F_{1}, \cdots, F_{n}$. Then $x \in U_{x} \subset$ $\bigcup_{i=1}^{n} F_{i}$. But by Lemma $2.7 \bigcup_{i=1}^{n} F_{i}$ is $M_{2}$ (or $M_{3}$ ), and then by Theorem 2.3 we see that $U_{x}$ is $M_{2}$ (or $M_{3}$ ). Now, since $X$ is paracompact and 
locally $M_{2}$ (or $M_{3}$ ), we get $X$ to be $M_{2}$ (or $M_{3}$ ) by Theorem 2.6, which completes the proof.

Whether Theorem 2.9 is true for $M_{1}$-space is unknown.

\section{Nagata spaces.}

Definition 3.1. A Nagata space $X$ is a $T_{1}$-space such that for each $x \in X$ there exist sequences of neighborhoods of $x,\left\{U_{n}(x)\right\}_{n=1}^{\infty}$ and $\left\{S_{n}(x)\right\}_{n=1}^{\infty}$, such that:

(1) for each $x \in X,\left\{U_{n}(x)\right\}_{n=1}^{\infty}$ is a local base of neighborhoods of $x$,

(2) for all $x, y \in X, S_{n}(x) \cap S_{n}(y) \neq \phi$ implies $x \in U_{n}(y)$.

The order pair $\left\langle\left\{U_{n}(x)\right\}_{n=1}^{\infty},\left\{S_{n}(x)\right\}_{n=1}^{\infty}\right\rangle$ is said to be a Nagata structure for $X$ if and only if, for each $x,\left\{U_{n}(x)\right\}_{n=1}^{\infty}$ and $\left\{S_{n}(x)\right\}_{n=1}^{\infty}$ are sequences of neighborhoods of $x$ satisfying the above two conditions.

Now having defined Nagata spaces, we get the following relation between a Nagata space and an $M_{3}$-space:

TheOREM 3.1. A topological space is a Nagata space if and only if it is first countable and $M_{3}$.

Proof. Let $X$ be a Nagata space with a Nagata structure $\left\langle\left\{U_{n}(x)\right\}_{n=1}^{\infty},\left\{S_{n}(x)\right\}_{n=1}^{\infty}\right\rangle$. Define $\boldsymbol{P}_{n}=\left\{\left(S_{n}(x)^{0}, U_{n}(x)\right): x \in X\right\}$ for each $n$. Then obviously $\bigcup_{n=1}^{\infty} \boldsymbol{P}_{n}$ is a pair-base. To show that each $\boldsymbol{P}_{n}$ is cushioned, we must show, for any index set $A$, that $\left(\cup\left\{S_{n}\left(x_{\alpha}\right)^{0}: \alpha \in A\right\}\right)^{-} \subset$ $\cup\left\{U_{n}\left(x_{\alpha}\right): \alpha \in A\right\}$. Suppose $y \notin \cup\left\{U_{n}\left(x_{\alpha}\right): \alpha \in A\right\}$. Then $S_{n}(y)^{0} \cap S_{n}\left(x_{\alpha}\right)^{0}=\phi$ for all $\alpha$ in $A$. Hence, $S_{n}(y)^{0} \cap\left(\cup\left\{S_{n}\left(x_{\alpha}\right)^{0}: \alpha \in A\right\}\right)=\phi$ and $y \notin$ $\left(\cup\left\{S_{n}\left(x_{\alpha}\right)^{0}: \alpha \in A\right\}\right)^{-}$. Thus $X$ is $M_{3}$ and first countable.

Now let $X$ be $M_{3}$ and first countable. For each $x \in X$, let $\left\{W_{n}(x)\right\}_{n=1}^{\infty}$ be a local base at $x$. Suppose $\bigcup_{n=1}^{\infty} P_{n}$ is a $\sigma$-cushioned pair-base for $X$. We can assume that for all $n,(X, X) \in \boldsymbol{P}_{n}$. For $m, n$ and $x \in X$ define

$$
U_{m, n}(x)=\cap\left\{\bar{P}_{2}: W_{m}(x) \subset P_{1}, P \in \boldsymbol{P}_{n}\right\}
$$

and

$$
S_{m, n}(x)=\cap\left\{P_{1}: W_{m}(x) \subset P_{1}, P \in \boldsymbol{P}_{n}\right\}-\cup\left\{\bar{P}_{1}: x \notin P_{2}, P \in \boldsymbol{P}_{n}\right\} .
$$

We wish to show that $\left\langle\left\{U_{m, n}(x)\right\}_{m, n=1}^{\infty},\left\{S_{m, n}(x)\right\}_{m, n=}^{\infty}=^{1}\right\rangle$ is a Nagata structure for $X$. Obviously $\left\{U_{m, n}(x)\right\}_{m, n=1}^{\infty}$ and $\left\{S_{m, n}(x)\right\}_{m, n=1}^{\infty}$ are sequences of neighborhoods of $x$ satisfying condition (1) in Definitition 3.1. To show (2), suppose $y \notin U_{m, n}(x)$. Then there exists a $P \in \boldsymbol{P}_{n}$ such that $W_{m}(x) \subset P_{1}$ and $y \notin \bar{P}_{2}$. Then, by definition of $S_{m, n}(x)$, we have $S_{m, n}(y) \cap \bar{P}_{1}=\phi$. But $S_{m, n}(x) \subset P_{1}$, so $S_{m, n}(x) \cap S_{m, n}(y)=\phi$, which completes the proof.

Now by virture of Theorem 3.1 and the fact subsets and countable products of first countable spaces are first countable, we obtain the results that: any subspace of a Nagata space is a Nagata space; a count- 
able product of Nagata spaces is Nagata; and in a Nagata space, separable $\longleftrightarrow$ Lindelöf $\longleftrightarrow$ the countable chain condition.

We can also get the following generalization (from $X$ being metric to $X$ being Nagata) of a well known extension theorem of Dugundji [3]:

THEOREM 3.2. Let $A$ be a closed subset of a Nagata space $X$ and let $f$ be a continuous map from $A$ into a convex subset $K$ of a locally convex topological linear space $Y$. Then $f$ can be extended to a continuous $g$ from $X$ to $K$.

Proof. Let $\left\langle\left\{U_{n}(x)\right\}_{n=1}^{\infty},\left\{S_{n}(x)\right\}_{n=1}^{\infty}\right\rangle$ be a Nagata structure for $X$. Without loss of generality we can suppose that, for $n<m$ and $y \in X$, we have $S_{m}(y) \subset S_{n}(y), U_{m}(y) \subset U_{n}(y)$, and $S_{1}(y)=U_{1}(y)=X$. Now for $x \in X-A$, put $n_{x}=\max \left\{n:\right.$ for some $\left.y \in A, x \in S_{n}(y)\right\}$ and $m_{x}=$ $\min \left\{n: U_{n}(x) \cap A=\phi\right\}$. By the paracompactness of $X-A$, let $\boldsymbol{V}$ be an open locally finite refinement of $\left\{S_{m_{x}}(x): x \in X-A\right\}$. For each $V \in \boldsymbol{V}$ pick $x_{V}$ such that $V \subset S_{m_{x_{V}}}\left(x_{V}\right)$, and pick $a_{V}$ such that $x_{V} \in S_{n_{x_{V}}}\left(a_{v}\right)$. Now let $\left\{p_{V}: V \in V\right\}$ be a partition of unity subordinate to $\boldsymbol{V}$, and define $g: X \rightarrow Y$ by

$$
g(x)=f(x) \quad \text { for } x \in A
$$

and

$$
g(x)=\sum_{V \in V} p_{V}(x) f\left(a_{V}\right) \quad \text { for } x \notin A .
$$

Then it can be shown without difficulty that $g$ is the desired extension of $f$.

4. Some metrization theorems. The following is a recent character:zation of metrizability by Nagata [13], which has the dual virture of being obviously satisfied by a metric space and of easily implying many other known metrization theorems. (The concept of a Nagata space was actually abstracted from this characterization.)

Theorem 4.1. (Nagata [13]). A $T_{1}$-space $X$ is metrizable if and only if $X$ is a Nagata space with a Nagata structure $\left\langle\left\{U_{n}(x)\right\}_{n=1}^{\infty},\left\{S_{n}(x)\right\}_{n=1}^{\infty}\right\rangle$ with the property that $x \in S_{n}(y)$ implies $S_{n}(x) \subset U_{n}(y)$ for all $x, y \in X$.

The following theorems are consequences of this result:

Theorem 4.2. A regular space $X$ is metrizable if and only if $X$ is an $M_{1}$-space with $\sigma$-closure preserving base $\boldsymbol{B}=\mathbf{U}_{n=1}^{\infty} \boldsymbol{B}_{n}$ such that, for each $x \in X$ and each $n, \cap\left\{B: x \in \boldsymbol{B}_{n}\right\}$ is neighborhood of $x$.

Proof. The sufficiency follows easily from Theorem 1.1. For the necessity, we put, for $x \in X$ and $m$, 


$$
U_{m}(x)=\bigcap\left\{\bar{B}: x \in B \in \boldsymbol{B}_{m}\right\},
$$

and

$$
S_{m}(x)=\bigcap\left\{B: x \in B \in \boldsymbol{B}_{m}\right\}-\bigcup\left\{\bar{B}: x \notin \bar{B} \text { and } B \in \boldsymbol{B}_{m}\right\} .
$$

Then it is easily checked that $\left\langle\left\{U_{m}(x)\right\}_{m=1}^{\infty},\left\{S_{m}(x)\right\}_{m=1}^{\infty}\right\rangle$ is a Nagata structure for $X$ with the property that $x \in S_{n}(y)$ implies $S_{n}(x) \subset U_{n}(y)$ for all $x, y \cong X$. Hence, according to Theorem 4.1, $X$ is metrizable.

CoRollary 4.3. A regular space $X$ is metrizable if and only if $X$ has a $\sigma$-closure preserving base $\boldsymbol{B}=\bigcup_{n=1}^{\infty} \boldsymbol{B}_{n}$ where each $\boldsymbol{B}_{n}$ is point finite.

Proof. The sufficiency follows from Theorem 1.1 and the necessity from Theorem 4.2.

The above theorem and corollary have analogues for the case of $M_{2}$ and $M_{3}$-spaces.

An interesting but unsolved problem poses itself here, namely: is an $M_{1}$-space with a $\sigma$-closure preserving base $\boldsymbol{B}=\bigcup_{n=1}^{\infty} \boldsymbol{B}_{n}$, where each $\boldsymbol{B}_{n}$ is point countable, necessarily metrizable?

We also have the following metrization theorem on $M_{1}$-spaces:

Theorem 4.4. (Bing [1]). A $T_{1}$-space $X$ is metrizable if and only if $X$ is an $M_{1}$-space with a $\sigma$-closure preserving base $\bigcup_{n=1}^{\infty} \boldsymbol{B}_{n}$ such that, for any $x \in X$ and open set $U$ containing $x$, there exists an $n$ such that $\phi \neq \bigcup \mathbf{U}\left\{B: x \in B \in \boldsymbol{B}_{n}\right\} \subset U$.

We can easily generalize this result to the following:

TheOREm 4.5. A $T_{1}$-space $X$ is metrizable if and only if $X$ is an $M_{3}$-space with a $\sigma$-cushioned pair-base $\bigcup_{n=1}^{\infty} \boldsymbol{P}_{n}$ with the property that for each $x \in X$ and open set $U$ containing $x$, there exists an $n$ such that $\phi \neq \bigcup\left\{P_{1} . x \in P_{1}, P \in \boldsymbol{P}_{n}\right\} \subset U$.

5. Completeness. According to Čech [2], a Hausdorff space is topologically complete if it is a $G_{\delta}$ in some compact Hausdorff space, and a Hausdorff space is completely metrizable if it has a compatible complete metric. Cech then proves that a metrizable space is completely metrizable if and only if it is topologically complete. In this section we investigate topologically complete $M_{i}$-spaces.

Theorem 5.1. (Nagata [13]). A topologically complete Nagata space is completely metrizable.

Actually Nagata's proof of Theorem 5.1 establishes the following result. 
THEOREM 5.2. Let $X$ be a xaracompact toxologically complete space, and suppose there exists a sequence of open converings $\left\{S_{n}\right\}_{n=1}^{\infty}$ such that, for every $x, y \in X, x \neq y$ implies there exists an $m$ such that $y \notin\left(\bigcup\left\{S: x \in S \in S_{m}\right\}\right)^{-}$. Then $X$ is completely metrizable.

We can generalize this result by virture of the following lemmas:

Lemma 5.3. Let $X$ be a paracompact space. Then, if there exists $a$ sequence of open coverings $\left\{\boldsymbol{V}_{n}\right\}_{n=1}^{\infty}$ such that $x \neq y$ implies there exists an $m$ such that $y \notin \bigcup\left\{V: x \in V \in V_{m}\right\}$, then there exists a sequence of open coverings $\left\{\boldsymbol{S}_{n}\right\}_{n=1}^{\infty}$ such that $x \neq y$ implies there exists an $m$ such that $y \notin\left(\bigcup\left\{S: x \in S \in S_{m}\right\}\right)^{-}$.

Proof. Let $\boldsymbol{W}_{m}$ be an open locally finite refinement of $\boldsymbol{V}_{m}$ such that, if $W \in \boldsymbol{W}_{m}$, then $\bar{W} \subset$ some $V \in \boldsymbol{V}_{m}$. For $V \in \boldsymbol{V}_{m}$, define $S_{V}=$ $\mathbf{U}\left\{W \in \boldsymbol{W}_{m}: \bar{W} \subset V\right\}$. Let $\boldsymbol{S}_{m}=\left\{S_{V}: V \in \boldsymbol{V}_{m}\right\}$. Then $\boldsymbol{S}_{m}$ is cushioned in $\boldsymbol{V}_{m}$ and in particular, if $x \notin \bigcup\left\{V \in \boldsymbol{V}_{m}: y \in V\right\}$, then $x \notin\left(\bigcup\left\{S_{V} \in \boldsymbol{S}_{m}: y \in V\right\}\right)^{-}$, and the conclusion of the lemma follows.

Lemma 5.4. The diagonal is a $G_{\delta}$ in $X \times X$ if and only if there exists a sequence of open coverings $\left\{S_{n}\right\}_{n=1}^{\infty}$ of $X$ such that for each $x, y \in X$ $x \neq y$ implies there exists an $m$ such that $y \notin \bigcup\left\{S: x \in S \in \boldsymbol{S}_{m}\right\}$.

Proof. Let $\Delta$ be the diagonal in $X \times X$. Suppose $\Delta=\bigcap_{n=1}^{\infty} G_{n}$ where each $G_{n}$ is open in $X \times X$. For each $n$, put $S_{n}=\{S: S$ open in $\left.X, S \times S \subset G_{n}\right\}$. Then if $x \neq y$, there exists an $m$ such that $(x, y) \notin G_{m}$ and hence $y \notin \bigcup\left\{S: x \in S \in S_{m}\right\}$.

Now assume we have such a sequence of open coverings $\left\{\boldsymbol{S}_{n}\right\}_{n=1}^{\infty}$. For each $n$, put $G_{n}=\bigcup\left\{S \times S: S \in S_{n}\right\}$. Then clearly $\Delta=\bigcap_{n=1}^{\infty} G_{n}$, which completes the proof.

Then obviously we can strengthen Theorem 5.2 to:

THEOREM 5.5. A paracompact topologically complete space whose diagonal is a $G_{\delta}$ in $X \times X$ is completely metrizable.

Now we generalize Theorem 5.1. to:

THEOREM 5.6. A topologically complete $M_{i}$-space is completely metrizable.

Proof. Let $X$ be an $M_{i}$-space. Then $X \times X$ is an $M_{i}$-space and thus perfectly normal; so the diagonal is a $G_{\delta}$. Now applying the previous theorem we complete the proof.

COROLlARY 5.7. A locally compact $M_{i}$-space is completely metrizable. 
Proof. It is well known that a locally compact space is open in any Hausdorff space in which it is densely embedded (Kelly [4], p. 163). Hence $X$ is open in $\beta(X)$, the Stone-Čech compactification of $X$, and, by Theorem 5.6, $X$ is completely metrizable.

Now we proceed to establish a "completeness-like" condition that will make a Nagata space topologically complete.

Definition 5.1. Let $X$ be a Nagata space. Then the Nagata structure $\left\langle\left\{U_{n}(x)\right\}_{n=1}^{\infty},\left\{S_{n}(x)\right\}_{n=1}^{\infty}\right\rangle$ is complete if, whenever $\left\{A_{n}\right\}_{n=1}^{\infty}$ is a decreasing sequence of nonempty closed sets such that for every $n$ there exists $x_{n}$ and $k_{n}$ such that $A_{k_{n}} \subset S_{n}\left(x_{n}\right)$, we have $\bigcap_{n=1}^{\infty} A_{n} \neq \phi$.

First we note without proof that:

THEOREM 5.8. Let $X$ be a Nagata space with Nagata structure $\left\langle\left\{U_{n}(x)\right\}_{n=1}^{\infty},\left\{S_{n}(x)\right\}_{n=1}^{\infty}\right\rangle$. Then the following are equivalent:

(1) $\left\langle\left\{U_{n}(x)\right\}_{n=1}^{\infty},\left\{S_{n}(x)\right]_{n=1}^{\infty}\right\rangle$ is complete.

(2) Whenever $\boldsymbol{A}$ is a family of closed sets having the finite intersection property such that for every $n$, there exists $A_{n} \in \boldsymbol{A}$ and $x_{n} \in X$ so that $A_{n} \subset S_{n}\left(x_{n}\right)$, then $\bigcap \boldsymbol{A} \neq \phi$.

(3) If $\left\{x_{m}\right\}_{m=1}^{\infty}$ is a sequence such that for any $n$ there exists $k_{n}, y_{n}$ such that $k_{n} \leqq m$ implies $x_{m} \subset S_{n}\left(y_{n}\right)$, then $\left\{x_{m}\right\}_{m=1}^{\infty}$ has a cluster point.

THEOREM 5.9. A Nagata space with a complete Nagata structure is completely metrizable.

Proof. For the proof, we need the concept of the Wallman compactification of a normal space (Wallman [18], Kelly [4, pp. 167-168]). Let $X$ be normal and let $\boldsymbol{F}$ be the family of all closed subsets of $X$. Define $w(X)$ to be the collection of all subfamilies of $\boldsymbol{F}$ which have the finite intersection property and are maximal with respect to this property. For $U$ open in $X$, we put $U^{+}=\{\boldsymbol{A} \in w(X)$ : for some $A \in \boldsymbol{A}, A \subset U\}$. Then $\left\{U^{+}: U\right.$ open in $\left.X\right\}$ is a base for some topology $\tau$. Then $\langle w(X), \tau\rangle$ is called the Wallman compactification of $X$. Then $w(X)$ is compact Hausdorff and $X$ is densely embedded in $w(X)$ by the homeomorphism $\phi(x)=\{A \in F: x \in A\}$.

To show that $X$ is completely metrizable we need only show that $X$ is a $G_{\delta}$ in $w(X)$. Let $\left\langle\left\{U_{n}(x)\right\}_{n=1}^{\infty},\left\{S_{n}(x)\right\}_{n=1}^{\infty}\right\rangle$ be the complete Nagata structure for $X$. For each $n$, put $G_{n}=\cup\left\{S_{n}(x)^{+}: x \in X\right\}$. Then $G_{n}$ is open and obviously $\phi(X) \subset \bigcap_{n=1}^{\infty} G_{n}$. Now suppose $\boldsymbol{A} \in \bigcap_{n=1}^{\infty} G_{n}$. Then for each $n$ there exists an $x_{n} \in X$ such that $\boldsymbol{A} \in S_{n}\left(x_{n}\right)^{+}$, which means that for each $n$ there exists $x_{n} \in X$ and $A_{n} \in \boldsymbol{A}$ so that $A_{n} \subset S_{n}\left(x_{n}\right)$. Hence by completeness $\bigcap \boldsymbol{A} \neq \phi$. So let $x \in \bigcap \boldsymbol{A}$, then since $\boldsymbol{A}$ is maximal with respect to the finite intersection property we must have $\boldsymbol{A}=$ $\phi(x) \in \phi(X)$. Hence, $\phi(X)=\bigcap_{n=1}^{\infty} G_{n}$, showing that $X$ is a $G_{\delta}$ in $w(X)$. 


\section{Semi-metric spaces.}

Definition 6.1. Let $d$ be a real-valued nonnegative function defined on $X \times X$. Then $d$ is a semi-metric for $X$ provided:

$$
\begin{aligned}
& d(x, y)=0 \text { if and only if } x=y, \\
& d(x, y)=d(y, x) \text { for all } x, y \in X .
\end{aligned}
$$

If $d$ is a semi-metric for $X$, the semi-metric topology is that determined by: $p$ is a limit point of $A \subset X$ if and only if inf $\{d(p, x): x \in A\}=0$. A topological space $\langle X, \tau\rangle$ is semi-metrizable if and only if there is a semi-metric $d$ such that the semi-metric topology agrees with $\tau$.

We can characterize semi-metric spaces as follows:

THEOREM 6.1. A Hausdorff space $X$ is semi-metrizable if and only if for all $x \in X$, there exists sequences of neighborhoods $\left\{U_{n}(x)\right\}_{n=1}^{\infty}$ and $\left\{S_{n}(x)\right\}_{n=1}^{\infty}$ such that $\left\{U_{n}(x)\right\}_{n=1}^{\infty}$ is a nested local base of neighborhoods of $x$, and for each $n$ and $x, y \in X, S_{n}(x) \subset U_{n}(x)$ and $y \in S_{n}(x)$ implies $x \in U_{n}(y)$.

Proof. For the sufficiency, put $S_{n}(x)=U_{n}(x)=\{y: d(x, y) \leqq 1 / n\}$. For the necessity, define $d(x, y)=\inf \left\{1 / n: x \in U_{n}(y)\right.$ and $\left.y \in U_{n}(x)\right\}$ where we assume $U_{1}(x)=X$ for all $x \in X$.

Now by virture of the preceding characterization of semi-metrizability, we obviously have:

\section{THEOREM 6.2. A Nagata space is semi-metrizable.}

McAuley [5] has given an example of a regular separable semimetric space $X$ which is not hereditarily sparable (that is, subsets are not necessarily separable). It follows by Theorems 2.3 and 2.5 that $X$ is not a Nagata space. In fact, it can be shown that $X$ is not even paracompact. An interesting unsolved problem is whether a paracompact (or even a regular Lindelöf) semi-metric space must be a Nagata space.

McAuley [5] has defined a semi-metric space to be strongly-complete if, whenever $\left\{A_{n}\right\}_{n=1}^{\infty}$ is a decreasing sequence of nonempty closed sets such that for every $n$ there exists $k_{n}$ and $x_{n}$ such that $A_{k_{n}} \subset\left\{y: d\left(x_{n}, y\right) \leqq 1 / n\right\}$, then we have $\bigcap_{n=1}^{\infty} A_{k_{n}} \neq \phi$. (Theorem 5.8 has an analogue for semimetric spaces). McAuley has proved the following result concerning strongly complete semi-metric spaces:

THEOREM 6.3. (McAuley [5]). A regular, hereditarily separable, strongly complete semi-metric space is metrizable.

The following two theorems, taken together, clarify and improve the above theorem of McAuley. 
THEOREM 6.4. A regular, hereditarily separable, semi-metric space is hereditarily Lindelöf (hence paracompact).

Proof. Let $\boldsymbol{U}$ be an open cover of $X$. For each $x \in X$, there exists $n_{x}$ and $U_{x} \in U$ such that $S_{n_{x}}(x)=\left\{y: d(x, y)<1 / n_{x}\right\} \subset U_{x}$. Put $A_{n}=$ $\left\{x \in X: n_{x}=n\right\}$. Then $A_{n}$ has a separable subset $\left\{d_{n}^{m}\right\}_{m=1}^{\infty}$ and it follows that $A_{n} \subset \bigcup_{m=1}^{\infty} S_{n}\left(d_{n}^{m}\right)$. Now choose $U_{n}^{m} \in \boldsymbol{U}$ such that $S_{n}\left(d_{n}^{m}\right) \subset U_{n}^{m}$. Then

$$
X=\bigcup_{n=1}^{\infty} A_{n} \subset \bigcup_{n, m=1}^{\infty} S_{n}\left(d_{n}^{m}\right) \subset \bigcup_{n, m=1}^{\infty} U_{n}^{m}
$$

So $\left\{U_{n}^{m}\right\}_{n, m=1}^{\infty}$ is a countable subcover of $\boldsymbol{U}$. So $X$ is Lindelöf and hence normal, but a normal semi-metric space is easily seen to be perfectly normal, and a perfectly normal Lindelöf space is easily seen to be hereditarily Lindelöf. So we conclude that $X$ is hereditarily Lindelöf and hence paracompact, which completes the proof.

THEOREM 6.5. A paracompact, strongly complete semi-metric space is completely metrizable.

Proof. Exactly analogously to the proof of Theorem 5.9 we show that $X$ is a $G_{\delta}$ in $w(X)$. Then we apply Lemma 5.4 and Theorem 5.5, where we take $\left.S_{m}=\left\{S_{m}(x)\right)^{0}: x \in X\right\}$ and $S_{m}(x)=\{y: d(x, y)<1 / m\}$, which completes the proof.

7. Closed continuous images. We have the following theorem about closed continuous images of metric spaces:

Theorem 7.1. (Stone [17], Morita and Hanai [11]). Let $f$ be $a$ closed continuous map of a metric space $X$ onto a topological space $Y$. Then the following are equivalent:

(1) $Y$ is first countable,

(2) for each $y \in Y$, the boundary of $f^{-1}(y), \partial f^{-1}(y)$, is compact,

(3) $Y$ is metrizable.

A special case of a closed continuous image of a space $X$ is $X / A$, the quotient space of $X$ formed by identifying the points of a closed subset $A$. Here, the natural map is clearly closed and continuous. Then, according to Theorem 7.1, if $X$ is a metric space and $A$ is a closed subset of $X$ with a non-compact boundary, then $X / A$ is not metrizable.

We have the following partial analogue to Theorem 7.1:

THEOREM 7.2. Let $X$ be an $M_{2^{-}}$(or $M_{3^{-}}$) space and $f$ a closed continuous function from $X$ onto any space $Y$. Then 
(1) if $Y$ is first countable, then for all $y \in Y, \partial f^{-1}(y)$ is compact,

(2) if for all $y \in Y, \partial f^{-1}(y)$ is compact, then $Y$ is $M_{2}$ (or $M_{3}$ ).

Proof. The proof of (1) is somewhat similar to Stone's proof of $(1) \rightarrow(2)$ in Theorem 7.1. To prove (2) for the $M_{2}$-case let $\bigcup_{n=1}^{\infty} \boldsymbol{B}_{n}$ be a $\sigma$-closure preserving quasi-base for $X$. Then $\bigcup_{n=1}^{\infty} A_{n}$ becomes a $\sigma$-closure preserving quasi-base for $Y$, where $\boldsymbol{A}_{n}=\left\{f\left(\bigcup_{i=1}^{k} A_{i}\right): A_{1}, \cdots, A_{k} \in \boldsymbol{B}_{n}\right\}$. The $M_{3}$-case is similar.

The converse of (1) is easily seen to be false by taking the identity map from a non-first countable $M_{2^{-}}$(or $M_{3^{-}}$) space onto itself. Also, Example 9.2 shows that the converse of (2) is false. It is unknown whether Theorem 7.2 is true for $M_{1}$-spaces.

It is also unsolved whether an arbitrary closed continuous image of an $M_{i}$-space is again $M_{i}$. However we can obtain the partial result that the quotient space of an $M_{2^{-}}$(or $M_{3}$ ) space with respect to a closed subset is again $M_{2}$ (or $M_{3}$ ).

For the $M_{2}$ case this result would follow if every closed subset $A$ of $X$ had a "local $\sigma$-closure preserving quasi-base" in the sense that there exists a $\boldsymbol{\sigma}$-closure preserving family $\boldsymbol{V}$ such that for every open $U$ containing $A, A \subset V^{0} \subset V \subset U$ for some $V \in \boldsymbol{V}$. For then, if $\boldsymbol{B}$ were a $\sigma$-closure preserving quasi-base for $X$, the image under the natural map of the family $\boldsymbol{V} \cup\{B \in \boldsymbol{B}: \bar{B} \cap A=\phi\}$ would be a $\sigma$-closure preserving quasi-base for $X \mid A$. As it turns out, we have the stronger result that every closed subset has a "local closure preserving quasi-base" as follows:

LeMma 7.3. Let $A$ be a closed subset of an $M_{2}$-space $X$. Then there exists a closure preserving family $\boldsymbol{V}$ of neighborhoods of $A$ such that for every open $U$ containing $A, A \subset V^{0} \subset V \subset U$ for some $V \in V$.

Proof. Let $\boldsymbol{B}=\bigcup_{n=1}^{\infty} \boldsymbol{B}_{n}$ be a $\sigma$-closure preserving quasi-base for $X$. Without loss of generality we can assume that the members of $B$ are closed and $\boldsymbol{B}_{n} \subset \boldsymbol{B}_{m}$ for $n<m$. For each $B \in \boldsymbol{B}_{n}$ we put

$$
R(B, n)=B-\bigcup\left\{W^{0}: A \cap W=\phi, W \in \boldsymbol{B}_{n}\right\} .
$$

Now let $\left\{\boldsymbol{S}_{\alpha}: \alpha \in E\right\}$ be the family of all subcollections of $\boldsymbol{B}$. For each $\alpha \in E$ and $n$, we put

$$
\begin{aligned}
V_{\alpha, n} & =\bigcup\left\{R(B, n): B \in\left(\boldsymbol{S}_{\alpha} \cap \boldsymbol{B}_{n}\right)\right\} \\
V_{\alpha} & =\bigcup_{n=1}^{\infty} V_{\alpha, n} \text { and } D=\left\{\alpha \in E: A \subset V_{\alpha}\right\} .
\end{aligned}
$$

To show $\boldsymbol{V}=\left\{V_{\alpha}: \alpha \in D\right\}$ is closure preserving, let $C \subset D$ and suppose $x \notin \bigcup\left\{\bar{V}_{\alpha}: \alpha \in C\right\}$. Then clearly $x \notin A$; so let $k$ be the least integer for which there exists a $B \in \boldsymbol{B}_{k+1}$ such that $x \in B^{0}$ and $B \cap A \neq \phi$. Then we have $V_{a_{n}} \cap B^{0}=\phi$ for every $n>k$ and $\alpha \in C$. Hence 
$x \notin\left(\bigcup\left\{V_{\alpha, n}: n>k, \alpha \in C\right\}\right)^{-}$. If $k \geqq 1$ (otherwise we are finished), then we also have $x \notin \cup\left\{W^{0}: A \cap W=\phi, W \in \boldsymbol{B}_{k}\right\}$. From the facts that $x \notin \bigcup\left\{W^{0}: A \cap W=\phi, W \in \boldsymbol{B}_{k}\right\}$ and $\quad x \notin \bigcup\left\{R(B, k): B \in\left(\boldsymbol{S}_{\alpha} \cap \boldsymbol{B}_{k}\right)\right\}$ it follows that $x \notin \bigcup\left(S_{\alpha} \cap \boldsymbol{B}_{k}\right)$. Since

$$
\left(\bigcup\left\{V_{\alpha m}: m \leqq k, \alpha \in C\right\}\right)^{-} \subset\left(\bigcup\left(\boldsymbol{S}_{\alpha} \cap \boldsymbol{B}_{k}\right)\right)^{-}=\mathbf{U}\left(\boldsymbol{S}_{\alpha} \cap \boldsymbol{B}_{k}\right)
$$

(because $\boldsymbol{B}_{k}$ is closure preserving), we have that $x \notin\left(\bigcup\left\{V_{a, n}: n \leqq k, \alpha \in C\right\}\right)^{-}$. Hence $x \notin\left(\bigcup\left\{V_{a}: \alpha \in C\right\}\right)^{-}$.

Finally, suppose $U$ is an open set containing $A$. For each $x \in A$ there exists $n_{x}$ and $B_{x} \in \boldsymbol{B}_{n_{x}}$ such that $x \in B_{x}^{0} \subset B_{x} \subset U$. Then $x$ is in the open set $B_{x}^{0}-\bigcup\left\{W: x \notin W, W \in \boldsymbol{B}_{n_{x}}\right\}$ which is included in $R\left(B_{x}, n_{x}\right)^{0}$. Hence $x \in R\left(B_{x}, n_{x}\right)^{0} \subset R\left(B_{x}, n_{x}\right) \subset U$. So putting $\boldsymbol{S}_{\alpha}=\left\{B_{x}: x \in A\right\}$ we clearly get $A \subset V_{\alpha}^{0} \subset V_{\alpha} \subset U$ with $\alpha \in D$, which completes the proof.

Lemma 7.4 has an analogue for $M_{3}$-spaces. Now by virtue of the remarks preceding Lemma 7.3 we clearly obtain:

THEOREM 7.4. Let $X$ be an $M_{2^{-}}$(or $M_{3^{-}}$) space and $A$ a closed subset of $X$. Then $X / A$ is $M_{2}$ (or $M_{3}$ ).

It is unknown whether the above theorem is true for $M_{1}$-spaces. However, we can get $X / A$ to be $M_{1}$ if $X$ is metrizable, as follows:

Lemma 7.5. Let $A$ be a closed subset of the metric space $X$. Then there exists a closure preserving family $\boldsymbol{V}$ of open sets such that for every open $U$ containing $A, A \subset V \subset U$ for some $V \in V$.

Proof. Let $\boldsymbol{B}=\bigcup_{n=1}^{\infty} \mathbf{B}_{n}$ be a $\sigma$-locally finite base for $X$ such that $\boldsymbol{B}_{n} \subset \boldsymbol{B}_{m}$ for $n<m$. For each $n$ put

$$
A_{n}=\{y \in X: \operatorname{dist}(y, A)<1 / n\} \text { and } \boldsymbol{A}_{n}=\left\{B \cap A_{n}: B \in \boldsymbol{B}_{n}\right\} \text {. }
$$

Then each $\boldsymbol{A}_{n}$ is locally finite. Let $\left\{\boldsymbol{W}_{\alpha}: \alpha \in D\right\}$ be the family of all subcollections of $\bigcup_{n=1}^{\infty} \boldsymbol{A}_{n}$ which cover $A$, and put $\boldsymbol{V}=\left\{V_{\alpha}: V_{\alpha}=\mathbf{U} \boldsymbol{W}_{\alpha}, \alpha \in D\right\}$. Then obviously for every open $U$ containing $A$ there exists $\alpha \in D$ such that $A \subset V_{\alpha} \subset U$. Now consider any $C \subset D$ and suppose $x \notin \bigcup\left\{\bar{V}_{\alpha}: \alpha \in C\right\}$. Then $x \notin A$ and there exists a $k$ such that $x \notin \bar{A}_{k}$; hence $\left(X-\bar{A}_{k}\right) \cap W=\phi$ for $W \in \boldsymbol{A}_{m} \cap \boldsymbol{W}_{\alpha}$ with $k \leqq m$ and $\alpha \in C$. Since $\bigcup_{i=1}^{k-1} \boldsymbol{A}_{i}$ is closure preserving, it follows that $x \in\left(\bigcup\left\{W \in \boldsymbol{A}_{m} \cap \boldsymbol{W}_{\alpha}: m<k, \alpha \in C\right\}\right)^{-}$. Then we get $x \notin\left(\bigcup\left\{V_{\alpha}: \alpha \in C\right\}\right)^{-}$, which completes the proof.

Now we obviously obtain the following:

Theorem 7.6. Let $X$ be a metric space and $A$ a closed subset of $X$. Then $X / A$ is $M_{1}$.

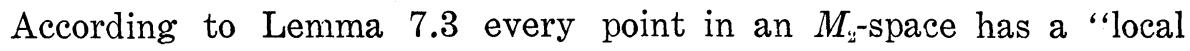


closure preserving quasi-base." It is unsolved, however, if every point in an $M_{1}$-space has a "local closure preserving base" (that is, an open local base which is closure preserving). Nevertheless, we can establish the following negative result:

Proposition 7.7. Suppose there exists an $M_{1}$-space $X$ with some point $p$ at which there does not exist a closure preserving open local base. Then

(1) there exists an $M_{2}$-space which is not $M_{1}$,

(2) there exists an $M_{1}$-space $Y$ with a closed subset $A$ such that $Y \mid A$ is not $M_{1}$.

Proof. Let $Y=\bigcup_{n=1}^{\infty} X_{n}$ where $n \neq m$ implies $X_{n} \cap X_{m}=\phi$ and each $X_{n}$ is homeomorphic to $X$ by a map $i_{n}$. Topologize $Y$ by: $O$ is open $\longleftrightarrow O \cap X_{n}$ is open in $X_{n}$ for all $n$. Let $p_{n}=i_{n}(p)$ and $A=$ $\left\{y \in Y: y=p_{n}\right.$ for some $\left.n\right\}$. Let $i$ be the natural map from $Y$ onto $Y / A$. Then clearly $A$ is closed and $Y$ is $M_{2}$; hence $Y \mid A$ is $M_{2}$. Now suppose $Y \mid A$ has a $\sigma$-closure preserving base $\boldsymbol{B}=\bigcup_{n=1}^{\infty} \boldsymbol{B}_{n}$. Then for each $n,\left\{i^{-1}(B) \cap X_{n}: A \in B \in \boldsymbol{B}_{n}\right\}$ is closure preserving in $X_{n}$. Hence, there exists an open $V_{n}$ in $X_{n}$ so that $p_{n} \in V_{n}$ and $A \in B \in \boldsymbol{B}_{n}$ implies $\left(i^{-1}(B) \cap X_{n}\right) \not \subset V_{n}$. Now put $V=\bigcup_{n=1}^{\infty} V_{n}$. Since $\boldsymbol{B}$ is a base there exists some $B$ in some $\boldsymbol{B}_{k}$ such that $A \in B \subset i(V)$, whence $\left(i^{-1}(B) \cap X_{k}\right) \subset V_{k}$, which is a contradiction. Hence, $Y \mid A$ is $M_{2}$ but not $M_{1}$.

8. The Topology of chunk-complexes. A chunk-complex is a topological space $\langle K, \tau\rangle$ having a family $\boldsymbol{K}$ of closed subsets, called chunks, such that

(1) $\bigcup K=K$,

(2) for $S, T \in K$, either $S \cap T=\phi$ or $S \cap T \in K$,

(3) for $S \in K,\{T \in K: T \subset S\}$ is finite,

(4) each $S \in \boldsymbol{K}$ is a compact metric space $\left\langle S, \rho_{S}\right\rangle$,

(5) $U \in \tau$ if and only if for every $S \in \boldsymbol{K}, S \cap U$ is open in $\left\langle S, \rho_{S}\right\rangle$.

If $\boldsymbol{B}$ is a collection of closed subsets of a space $X$, then $\boldsymbol{B}$ dominates $X$ provided that, for every subfamily $\boldsymbol{A}$ of $\boldsymbol{B}$, if $C \subset \cup \boldsymbol{A}$ and $A \cap C$ is closed in $A$ for all $A \in \boldsymbol{A}$, then $C$ is closed in $X$.

Theorem 8.1. (Michael [7, pp. 379-380], Morita [10]). If $X$ is dominated by a collection of paracompact (or perfectly normal) subsets, then $X$ is paracompact (or perfectly normal).

Using Theorem 8.1, it is easy to show that.

Lemma 8.2. A chunk-complex is dominated by the set of its chunks, and hence is paracompact and perfectly normal. 
In this section we establish the stronger result that each chunkcomplex is an $M_{1}$-space.

For the proof we establish the following notation: For $S \in \boldsymbol{K}$ define $\Delta(S)=\{T \in \boldsymbol{K}: T \subset S, T \neq S\}$. Define $\boldsymbol{K}_{0}=\{S \in \boldsymbol{K}: \Delta(S)=\phi\}$ and, assuming $\boldsymbol{K}_{m}$ has been defined for $0 \leqq m<n$, we define

$$
\boldsymbol{K}_{n}=\left\{S \in \boldsymbol{K}: \Delta(S) \subset \bigcup_{i=0}^{n-1} \boldsymbol{K}_{i}\right\}-\bigcup_{i=0}^{n-1} \boldsymbol{K}_{i} .
$$

Then $\bigcup_{n=1}^{\infty} \boldsymbol{K}_{n}=\boldsymbol{K}$, by induction on the number of subchunks. For $S \in \boldsymbol{K}$ put $\partial S=\bigcup(\Delta(S)), S^{0}=S-\partial S$, and $\boldsymbol{A}_{S}=\{T \in \boldsymbol{K}: S \subset T\}$. Then obviously $\bigcup\left\{S^{0}: S \in \boldsymbol{K}\right\}=K$. Let $N$ be the set of nonnegative integers and $M=\{1 / n: n \in N-\{0\}\}$.

Theorem 8.3. A chunk-complex is an $M_{1}$-space.

Proof. Let $\langle K, \tau\rangle$ be a chunk-complex with a set of chunks $\boldsymbol{K}$. First we observe that for each $n \in N, P \in \boldsymbol{K}_{n}$, there exists a countable family $\boldsymbol{B}(P)=\left\{P_{m}: m \in N\right\}$ of open sets in $P^{0}$ forming a base for points in $P^{0}$ so that $\bar{P}_{m} \in P^{0}$ for all $m \in N$. Fix $n \in N, P \in \boldsymbol{K}_{n}$ and $B \in \boldsymbol{B}(P)$. Let $g: \boldsymbol{A}_{P} \rightarrow M$. Then we define a candidate $B_{g}$ for our base as follows: By normality, let $W$ be an open set containing $\bar{B}$ and such that $\bar{W} \cap\left(\bigcup\left\{T \in \boldsymbol{K}: T \cap P^{0}=\phi\right\}\right)=\phi$. Now, by induction, for any $T \in \boldsymbol{K}_{n} \cap \boldsymbol{A}_{P}$ we necessarily have $T=P$ and we define $B_{g}^{P}=B$ and $\dot{B}_{g}^{P}=\phi$. Now assume we have defined $B_{g}^{S}$ for all $S \in \boldsymbol{K}_{n+k} \cap \boldsymbol{A}_{P}$ with $k<m$. Then for any $T \in \boldsymbol{K}_{n+m} \cap \boldsymbol{A}_{P}$ we put

$$
\dot{B}_{g}^{r}=\bigcup\left\{B_{g}^{S}: S \in \Delta(T) \cap \boldsymbol{A}_{P}\right\}
$$

and

$$
B_{g}^{T}=W \cap\left\{y \in T: \rho_{T}\left(\dot{B}_{g}^{T}, y\right)<\min \left[g(T), \rho_{T}\left(y, \partial T-\dot{B}_{g}^{T}\right)\right]\right\} .
$$

Finally we put

$$
B_{g}=\bigcup\left\{B_{g}^{T}: T \in \boldsymbol{A}_{P}\right\} .
$$

We note that for all $T \in \boldsymbol{A}_{P}$ we have $\left(B_{g}^{T} \cap \partial T\right) \subset \dot{B}_{g}^{T},\left(\left(B_{g}^{T}\right)^{-} \cap \partial T\right) \subset\left(\dot{B}_{g}^{T}\right)^{-}$, and if $S \notin \boldsymbol{A}_{P},\left(B_{g}^{T}\right)^{-} \cap S=\phi$.

Now we need to establish the following lemma:

Lemma 8.4. For all $P \in \boldsymbol{K}_{n}$ and $S, T \in \bigcup_{k=0}^{m} \boldsymbol{K}_{n+k} \cap \boldsymbol{A}_{P}$,

(a) $\dot{B}_{g}^{S}$ is open in $\partial S$,

(b) $\dot{B}_{g}^{s} \subset B_{g}^{s}$,

(c) $\left(B_{g}^{S} \cap T\right) \subset B_{g}^{T}$,

(d) $\left(\left(B_{g}^{S}\right)^{-} \cap T\right) \subset\left(B_{g}^{T}\right)^{-}$.

Proof. By induction on $m$ : if $m=0$, then $S=T=P$ and all conditions are obviously satisfied. Now assume that (a), (b), (c) and (d) hold 
for all $k<m$, and let us prove this for $m$.

(a) Applying the induction hypothesis on (c) we get for all $R, Q \in \Delta(S) \cap \boldsymbol{A}_{P}$ that $\left(B_{g}^{R} \cap Q\right) \subset B_{g}^{Q}$. Hence

$$
\partial S-\dot{B}_{g}^{S}=\partial S-\bigcup\left\{B_{g}^{T} \in \Delta(S)\right\}=\bigcup\left\{R-B_{g}^{R}: R \in \Delta(S)\right\} .
$$

But each $R-B_{g}^{R}$ is closed in $R$ which is in turn closed in $\partial S$. Hence $\partial S-\dot{B}_{g}^{S}$ is closed in $\partial S$ for $S \in \boldsymbol{K}_{n+m}$.

(b) Then if $y \in \dot{B}_{g}^{S}, \rho_{S}\left(y, \dot{B}_{q}^{S}\right)=0$ and $\rho_{S}\left(y, S-\dot{B}_{g}^{S}\right)>0$, so $y \in B_{g}^{S}$. Hence we have $\dot{B}_{g}^{S} \subset B_{g}^{S}$ for all $S \in \boldsymbol{K}_{n+m}$.

(c) If $S \not \subset T$, then $\left(B_{g}^{S} \cap T\right) \subset\left(B_{g}^{S} \cap(T \cap S)\right) \subset\left(B_{g}^{S} \cap \partial S\right) \subset \dot{B}_{g}^{S}$. So if $x \in B_{g}^{S} \cap T$, then $x \in$ some $B_{g}^{R}$ with $R \in \Delta(S)$, and then $x=\left(B_{g}^{R} \cap(T \cap S)\right) \subset B^{T \cap S}$ by the induction hypothesis on (c). If $S \cap T=T$, then $B_{g}^{S \cap T}=B_{g}^{T}$. If $S \cap T \neq T$, then $S \cap T \in \Delta(T)$, and by (b) we have $B_{g}^{S \cap T} \subset B_{g}^{T}$. Hence if $S \not \subset T,\left(B_{g}^{S} \cap T\right) \subset B_{g}^{T}$. If $S \subset T$, then $B_{g}^{S} \subset B_{g}^{T}$ by (b). Hence $\left(B_{g}^{S} \cap T\right) \subset B_{g}^{T}$ for all $S, T \in \boldsymbol{K}_{n+m} \cap \boldsymbol{A}_{P}$.

(d) The proof of (d) is exactly similar to (c) above; but here we use the fact that $\left(\left(B_{g}^{S}\right)^{-} \cap S\right) \subset\left(\dot{B}_{g}^{S}\right)^{-}$.

This completes the proof of Lemma 8.4.

For $m, n \in N, P \in \boldsymbol{K}_{n}$, define $\boldsymbol{V}_{P}^{m}=\left\{\left(P_{m}\right)_{g}: g: \boldsymbol{A}_{P} \rightarrow M\right\}$ and $\boldsymbol{U}_{n}^{n}=$ $\bigcup\left\{\boldsymbol{V}_{P}^{m}: P \in \boldsymbol{K}_{n}\right\}$. Now we will show that

(a) each $\left(P_{m}\right)_{g}$ is open,

(b) $\bigcup\left\{V_{P}^{m}: m \in N\right\}$ is a base for points in $P^{0}$,

(c) each $V_{P}^{m}$ is closure preserving,

(d) each $\boldsymbol{U}_{n}^{m}$ is closure preserving.

Then, since $\bigcup\left\{P^{0}: P \in \bigcup_{n=1}^{\infty} \boldsymbol{K}_{n}\right\}=K, \boldsymbol{B}=\bigcup\left\{\boldsymbol{U}_{n}^{m}: n, m \in N\right\}$ will be the desired $\sigma$-closure preserving base for $K$.

(a) each $\left(P_{m}\right)_{g}$ is open. Let $P_{m}=B$. It then suffices to show that for every $S \in \boldsymbol{A}_{p}, S \cap B_{g}$ is open in $S$. But by Lemma 8.4, $S \cap B_{g}=$ $\bigcup\left\{S \cap B_{g}^{T}: T \in \boldsymbol{A}_{P}\right\}=S \cap B_{g}^{S}$, which is open in $S$ by construction.

(b) $\bigcup\left\{\boldsymbol{V}_{P}^{m}: m \in N\right\}$ is a base for points in $P^{0}$. Let $P \in \boldsymbol{K}_{n}, x \in P^{0}$, and $U$ be on open set containing $x$. Choose $B \cong \boldsymbol{B}(P)$ such that $x \in B \subset$ $\bar{B} \subset\left(U \cap P^{0}\right)$. We want to find $g: \boldsymbol{A}_{P} \rightarrow M$ so that $x \in B_{g} \subset U$. By induction on $m$, we define $g(T)$ for $T \in \boldsymbol{K}_{n+m} \cap \boldsymbol{A}_{P}$ so that $\left(B_{g}^{T}\right)^{-} \subset U$. For $m=0$ we have $T=P$ and $\left(B_{g}^{T}\right)^{-}=\bar{B} \subset(P \cap U)$ for any $g: \boldsymbol{A}_{P} \rightarrow M$, so put $g(P)=1$. Now assume we have defined $g(S)$ for every $S \in \boldsymbol{K}_{n+k} \cap \boldsymbol{A}_{P}$ with $k<m$ so that $\left(B_{g}^{S}\right)^{-} \subset U$. Let $T \in \boldsymbol{K}_{n+m} \cap \boldsymbol{A}_{P}$. Then, by the induction hypothesis, $\left(\dot{B}_{g}^{T}\right)^{-}=\bigcup\left\{\left(B_{g}^{S}\right)^{-}: S \in \Delta(T)\right\} \subset(U \cap T)$. So by the compactness of $T$ there exists $\beta \in M$ so that $\left\{y \in T: \rho_{T}\left(y, \dot{B}_{g}^{T}\right) \leqq\right.$ $\beta\} \subset(T \cap U)$. Then put $g(T)=\beta$. Then we have

$$
\begin{aligned}
\left(B_{g}^{T}\right)^{-}= & \left(W \cap\left\{y \in T: \rho_{T}\left(y, \dot{B}_{g}^{T}\right)<\min \left[g(T), \rho_{T}\left(y, \partial T-\dot{B}_{g}^{T}\right)\right]\right\}\right)^{-} \\
& \subset\left\{y \in T: \rho_{T}\left(y, \dot{B}_{g}^{T}\right) \leqq g(T)\right\} \subset(T \cap U) .
\end{aligned}
$$

Hence $x \in B_{g}=\bigcup\left\{B_{g}^{T}: T \in \boldsymbol{A}_{P}\right\} \subset U$, with $B_{g} \in \boldsymbol{V}_{P}^{m}$ and $B=P_{m}$. 
(c) each $\boldsymbol{V}_{P}^{m}$ is closure-preserving. First we need the following result:

Lemma 8.5. (Michael [8]). Let $D=\prod_{i=1}^{j} M_{i}$, where $M_{i}=M$ for all $i$. For all $x, y \in D$, define $x \leqq y$ if and only if $x_{i} \leqq y_{i}$ for all $i$. Then $\langle D, \leqq$ is a partially ordered set with the property that, for each $C \subset D$, there exist $c_{1}, \cdots c_{m} \in C$ so that, for all $c \in C$, there exists $c_{k}(1 \leqq k \leqq m)$ such that $c \leqq c_{k}$.

Now let $\left\{B_{g}: g \in G\right\}$ be a subfamily of $V_{P}^{m}$ with $P_{m}=B$. For every $T \in \boldsymbol{A}_{P}$ we must show $T \cap\left(\bigcup\left\{\bar{B}_{g}: g \in G\right\}\right)$ is closed. First we show that $\bar{B}_{g}=\bigcup\left\{\left(B_{g}^{S}\right)^{-}: S \in \boldsymbol{A}_{P}\right\}$. For this it suffices to show, for every $T \in \boldsymbol{A}_{P}$, that $\left.T \cap\left(\bigcup\left\{B_{g}^{S}\right)^{-}: S \in \boldsymbol{A}_{P}\right\}\right)$ is closed. But by part (d) of Lemma 8.4, $T \cap\left(\bigcup\left\{\left(B_{g}^{S}\right)^{-}: S \in \boldsymbol{A}_{P}\right\}\right)=\left(B_{g}^{T}\right)^{-}$. Then

$T \cap\left(\bigcup\left\{\bar{B}_{g}: g \in G\right\}\right)=T \cap\left(\bigcup\left\{\left(B_{g}^{S}\right)^{-}: g \in G, S \in \boldsymbol{A}_{P}\right\}\right)=\bigcup\left\{\left(B_{g}^{T}\right)^{-}: g \in G\right\}$.

Now we apply Lemma 8.5 above to the subset $A=\left\{\left(g\left(S_{1}\right), \cdots, g\left(S_{k}\right)\right): g \in G\right\}$ of the partially ordered set $\prod_{i=1}^{k} M_{i}$, where $\left\{S_{1}, \cdots, S_{k}\right\}=\Delta(T) \cap \boldsymbol{A}_{P}$. Notice that, if $g\left(S_{i}\right) \leqq h\left(S_{i}\right)$ for all $i$ with $g, h \in G$, then we have $\left(B_{g}^{T}\right)^{-} \subset\left(B_{h}^{T}\right)^{-}$. Hence by Lemma 8.5 we get $g_{1}, \cdots, g_{n} \in G$ such that

$$
T \cap\left(\bigcup\left\{\bar{B}_{g}: g \in G\right\}\right)=\bigcup\left\{\left(B_{g}^{T}\right)^{-}: g \in G\right\}=\bigcup_{i=1}^{n}\left\{\left(B_{g_{i}}^{T}\right)^{-}\right\},
$$

which is closed.

(d) each $\boldsymbol{U}_{n}^{m}$ is closure preserving. Let $\boldsymbol{U}$ be a subfamily of $\boldsymbol{U}_{n}^{m}$. Then we can express $\boldsymbol{U}$ as $\left\{\left(P_{m}\right)_{g}: g \in G_{P}, P \in \boldsymbol{P}\right\}$ for some $\boldsymbol{P} \subset \boldsymbol{K}_{n}$ and $G_{P} \subset\left\{g: g: \boldsymbol{A}_{P} \rightarrow M\right\}$. Let $T \in \boldsymbol{K}$. If $P \not \subset T$, then $T \notin \boldsymbol{A}_{P}$ and $\left(\left(P_{m}\right)_{g}\right)^{-} \cap T=\phi$. But there are only finitely many $P \in P$ contained in $T$. Hence there exist $P^{1}, \cdots, P^{k} \in \boldsymbol{P}$ so that

$$
T \cap\left(\bigcup\left\{\bar{B}_{g}: B_{g} \in U\right\}\right)=T \cap\left(\bigcup\left\{\left(\left(P_{m}^{i}\right) g\right)^{-}: 1 \leqq i \leqq k, g \in G_{P i}\right\}\right)
$$

which is closed by part (c) above.

This completes the proof of the theorem.

Corollary 8.6. A $C W$-complex (Whitehead [19]) is an $M_{1}$-space.

Proof. Let $\langle K, \tau\rangle$ be a $C W$-complex. Then the family of finite subcomplexes is a family of chunks, whence the $C W$-complex $\langle K, \tau\rangle$ is $M_{1}$. (See Whitehead [19] for terminology).

CoRollary 8.7. A countable product of $C W$-complexes is an $M_{\mathrm{i} \text { - }}$ space; hence; both paracompact and perfectly normal.

Proof. Apply Theorems 2.2 and 2.4 and Corollary 8.6.

9. Some examples. In the sequel, $R$ will denote the real numbers 
and $N$ the natural numbers. We will also use the notation $\langle x, y\rangle$ for the point $(x, y) \in R \times R$ to distinguish it from $(s, t)$ which will mean the open interval $\{x \in R: s<x<t\}$ and [s,t] which will be the closed interval $\{x \in R: s \leqq x \leqq t\}$.

ExAmple 9.1. A non-metrizable first countable $M_{1}$-space.

Let $R^{\prime}$ be the rational numbers. For $x \in R$, put $L_{x}=\{\langle x, y\rangle:\langle x, y\rangle €$ $R \times R, 0<y\}$ and $X=R \cup\left(\bigcup\left\{L_{x}: x \in R\right\}\right)$. Then we will define a base for $X$ as follows: For $s, t \in R^{\prime}$ and $z=\langle x, w\rangle \in L_{x}$ such that $0<s<w<t$ we put $\bigcup_{s . t}^{x}(z)=\{\langle x, y\rangle: S\langle y<t\}$ and let $\boldsymbol{U}$ be the set of all such $U_{s, t}^{x}(z)$. For $r, s, t \in R^{\prime}$ and $z \in R$ such that $s<z<t$ and $r>0$, we put

$$
V_{r, s, t}(z)=(s, t) \cup(\bigcup\{\langle w, y\rangle: 0<y<r, w \in(s, t)-\{z\}\}),
$$

and let $\boldsymbol{V}$ be the set of all such $V_{r, s, t}(z)$. Now put $\boldsymbol{B}=\boldsymbol{U} \cup \boldsymbol{V}$. Then it can be easily shown that $\boldsymbol{B}$ is a $\sigma$-closure preserving base making $X$ into a non-metrizable first countable $M_{1}$-space.

The following example is more powerful than Example 9.1. But here the proof of $M_{1}$-ness, which is due to Jun-iti Nagata, is far from being straightforward. (The space of the example seems to have first appeared in McAuley [5]; Nagata [13] gives it without proof of $M_{1}$-ness as an example of a non-metrizable, separable Nagata space.)

EXAMPLE 9.2. [Nagata]. A non-metrizable, separable, first countable $M_{1}$-space.

Let $X=\{\langle x, y\rangle:\langle x, y\rangle \in R \times R, 0<x<1,0 \leqq y\}$. Clearly $X-(0,1)$, as a subset of $R \times R$, has a $\sigma$-closure preserving base $\boldsymbol{B}$. For $n \in N$ and $\langle p, 0\rangle \in X$, we define

$$
U_{n}(p)=\{p\} \cup\left\{\langle x, y\rangle \in X: y<n-\left(n^{2}-(x-p)^{2}\right)^{1 / 2},|x-p|<1 / n\right\} .
$$

Then $\boldsymbol{B} \cup\left\{U_{n}(p): n \in N,\langle p, 0\rangle \in X\right\}$ is a base which clearly generates a regular topology. Obviously $X$ is separable, first countable, and not second countable; hence $X$ is not metrizable.

To show the existence of a $\sigma$-closure preserving base for $X$, it suffices to show one for points in $(0,1)$. For $m, q \in N, m<q$, and $0 \leqq k \leqq 2^{m+1}-2$, we define

$$
W_{q, m, k}=\left\{\langle x, y\rangle:(k) 2^{-m-1}<x<(k+2) 2^{-m-1}, 0<y \leqq 2^{-q}\right\} .
$$

Now consider any $U_{n}(p)$. Then we can choose $m, k \in N$ so that

$$
(k) 2^{-m-1}<n^{-1}+p \text { and }(k-4) 2^{-m-1} \leqq p<(k-3) 2^{-m-1} \text {. }
$$

For this $m, k$, we put

$$
q=\min \left\{j: W_{j, m, k-2} \subset U_{n}(p)\right\},
$$




$$
\begin{aligned}
& I_{1}=W_{q, m, k-2}, \\
& a_{1}=(k) 2^{-m-1}, \\
& a_{2}=(k-2) 2^{-m-1}, \\
& b_{1}=2^{-q} .
\end{aligned}
$$

Now for each $i \in N$, we choose $k_{i}$ so that

$$
\left(k_{i}-4\right) 2^{-m-i-1} \leqq p<\left(k_{i}-3\right) 2^{-m-i-1} .
$$

Then we put

$$
\begin{aligned}
q_{i} & =\min \left\{j: W_{i, m+i, k_{i}-2} \subset U_{n}(p)\right\}, \\
I_{i+1} & =W_{q_{i}, m+i, k_{i}-2}, \\
a_{i+2} & =\left(k_{i}-2\right) 2^{-m-i-1}, \\
b_{i+1} & =2^{-q_{i}} .
\end{aligned}
$$

Now it follows that for each $i, j \in N, i<j$ implies $a_{j}<a_{i}$ and $b_{j}<b_{i}$, and obviously $b_{i} \rightarrow 0$ and $a_{i} \rightarrow p$.

We also choose $m^{\prime}, k^{\prime} \in N$ such that

$$
p-n^{-1}<\left(k^{\prime}\right) 2^{-m^{\prime}-1} \text { and }\left(k^{\prime}+3\right) 2^{-m^{\prime}-1}<p \leqq\left(k^{\prime}+4\right) 2^{-m^{\prime}-1} .
$$

Then we put

$$
\begin{aligned}
& q^{\prime}=\min \left\{j: W_{j, m^{\prime}, k^{\prime}} \subset U_{n}(p)\right\}, \\
& I_{1}^{\prime}=W_{q^{\prime}, m^{\prime}, k^{\prime}}, \\
& a_{1}^{\prime}=\left(k^{\prime}\right) 2^{-m^{\prime}-1}, \\
& a_{2}^{\prime}=\left(k^{\prime}+2\right) 2^{-m^{\prime}-1}, \\
& b_{1}^{\prime}=2^{-q^{\prime}} .
\end{aligned}
$$

Now for $i \in N$, we choose $k_{i}^{\prime}$ so that

$$
\left(k_{i}^{\prime}+3\right) 2^{-m^{\prime}-i-1}<p \leqq\left(k_{i}^{\prime}+4\right) 2^{-m^{\prime}-i-1} .
$$

Then put

$$
\begin{aligned}
q_{i}^{\prime} & =\min \left\{j: W_{j, m^{\prime}+i, k_{i}^{\prime}} \subset U_{n}(p)\right\}, \\
I_{i+1}^{\prime} & =W_{q_{i}^{\prime}, m+i, k_{i}}, \\
a_{i+2}^{\prime} & =\left(k_{i}^{\prime}+2\right) 2^{-m^{\prime}-i-1}, \\
b_{i+1}^{\prime} & =2^{-q_{i}^{\prime}} .
\end{aligned}
$$

Then for each $i, j \in N, i<j$ implies $a_{i}^{\prime}<a_{j}^{\prime}$ and $b_{i}^{\prime}<b_{j}^{\prime}$, and obviously $b_{i}^{\prime} \rightarrow 0$ and $\alpha_{i}^{\prime} \rightarrow p$.

Now putting

$$
N_{n}(p)=\left(\left(\left(\bigcup_{j=1}^{\infty} I_{j}\right) \cup\left(\bigcup_{j=1}^{\infty} I_{j}^{\prime}\right)\right)^{-}\right)^{0},
$$

it can be shown that $p \in N_{n}(p) \subset U_{n}(p)$. 
Now consider the countable set

$$
T=\left\{\left\langle\left(k^{\prime}\right) 2^{-m^{\prime}},(k) 2^{-m}\right\rangle: k, k^{\prime}, m, m^{\prime} \in N,\left(k^{\prime}\right) 2^{-m^{\prime}}<(k) 2^{-m}\right\} .
$$

For $t=\left\langle\left(k^{\prime}\right) 2^{-m^{\prime}},(k) 2^{-m}\right\rangle \in T$, put

$$
\boldsymbol{B}_{t}=\left\{N_{n}(p): a_{1}^{\prime}=\left(k^{\prime}\right) 2^{-m^{\prime}}, a_{1}=(k) 2^{-m}\right\} .
$$

Then obviously $\bigcup\left\{\boldsymbol{B}_{t}: t \in T\right)=\left\{N_{n}(p): n \in N, p \in(0,1)\right\}$, which is a base for points in $(0,1)$. Finally, it can be shown that each $\boldsymbol{B}_{t}$ is closure preserving. Hence $\bigcup\left\{\boldsymbol{B}_{t}: t \in T\right\}$ is a $\sigma$-closure preserving base and $X$ is an $M_{1}$-space.

If $X$ is the space in Example 9.2, then it can be shown without difficulty that $X /(0,1)$ is an $M_{1}$-space with $(0,1)$ having a closure preserving local base.

EXAMPLE 9.3. There exists a non-metrizable $M_{1}$-space $X$ with $p \in X$ such that $p$ has an uncountable closure preserving local base and $X-\{p\}$ is homeomorphic to $R$.

Let $p \notin R$ and put $X=R \cup\{p\}$. Let $\left\{r_{n}\right\}_{n=1}^{\infty}$ be an enumeration of the integers and put $B=\{1 / n: n \in N-\{1\}\} \cup\{0\}$. Let $F$ be the set of all functions from the integers $I$ to $B$ such that either there exists $r \in I$ such that if $s<r$, then $f(s)=0$ and if $r \leqq s$, then $f(s) \neq 0$; or for all $r \in I, f(r) \neq 0$. For $f \in F$, put $U_{f}=\bigcup_{n=1}^{\infty}\left(r_{n}-f\left(r_{n}\right), r_{n}+f\left(r_{n}\right)\right)$ where if $f\left(r_{n}\right)=0,\left(r_{n}, r_{n}\right)=\phi$. Let $\boldsymbol{U}=\left\{\{p\} \cup U_{f}: f \in F\right\}$ and $\boldsymbol{B}$ be a countable base for $R$. Then it is obvious that $\boldsymbol{U} \cup \boldsymbol{B}$ is a $\sigma$-closure preserving base for $X$. Moreover, it is easy to see that $X$ is not first countable at $p$ and $R$ is homeomorphic to $X-\{p\}$.

It is clear that this construction can be carried out for any noncompact metric space without isolated points. In particular, carrying it out for the rational numbers we get a countable non-metrizable $M_{1}$-space.

EXAMPLE 9.4. (Michael [9]). We can get another countable nonmetrizable $M_{1}$-space by taking the subspace $I \cup\{p\}$ of $\beta(I)$, where $I$ is the integers and $\beta(I)$ is the Stone-Čech compactification of $I$ and $p \in \beta(I)-I$. Here the family of all open sets containing $p$ is closure preserving.

\section{BIBLIOGRAPHY}

1. R. H. Bing, Metrization of topological spaces, Canadian J. Math., 3 (1951), 175-186.

2. E. Cěch, On bicompact spaces, Ann. Math., 38 (1937), 823-844.

3. J. Dugundji, An extension of Tietze's theorem, Pacific J. Math., 1 (1951), 353-367.

4. J. L. Kelley, General Topology, New York, Van Nostrand Co., Inc., 1955.

5. L.F. McAuley, A relation between perfect separability, completeness and normality in semi-metric spaces, Pacific J. Math., 6 (1956), 315-326.

6. E. A. Michael, Yet another note on paracompact spaces, Proc. Amer. Math. Soc., 10 (1959), 309-314. 
7. , Continuous selection I, Ann. Math., 63 (1956), 361-382.

8. - On a class of partially ordered sets, Amer. Math. Monthly, 67 (1960), 448449.

9. —, Another note on paracompact spaces, Proc. Amer. Math. Soc., 8 (1957), 822828.

10. K. Morita, On spaces having the weak topology with respect to closed coverings, II, Proc. Japan Acad., 30 (1954), 711-717.

11. _ and S. Hanai, Closed mappings and metric spaces, Proc. Japan Acad., 32 (1956), 10-14.

12. J. Nagata, On a necessary and sufficient condition of metrizability, J. Inst. Polytech., Osaka City Univ., 1 (1950), 93-100.

13. - A contribution to the theory of metrization, J. Inst. Polytech., Osaka City Univ., 8 (1957), 185-192.

14. Yu. M. Smirnov, A necessary and sufficient condition for metrizability of a topological space, Doklady Akad. Nauk S. S. S. R., (N. S.), 77 (1951), 197-200.

15. - On metrization of topological spaces, Uspehi Matem. Nauk, 6 (1951), 100-111. 16. R. H. Sorgenfrey, On the topological product of paracompact spaces, Bull. Amer. Math. Soc., 53 (1947), 631-632.

17. A. H. Stone, Metrizability of decomposition spaces, Proc. Amer. Math. Soc., 7 (1956), 690-700.

18. H. Wallman, Lattices and topological spaces, Ann. Math., 39 (1938), 112-126.

19. J. H. C. Whitehead, Combinatorial homotopy, I, Bull. Amer. Math. Soc., 55 (1949), 213245.

UNIVERSITY OF CALIFORNIA, SANTA BARBARA 



\section{PACIFIC JOURNAL OF MATHEMATICS}

\section{EDITORS}

Ralph S. Phillips

Stanford University

Stanford, California

F. H. BrownelL

University of Washington

Seattle 5 , Washington
A. L. Whiteman

University of Southern California Los Angeles 7. California

L. J. PAIGE

University of California

Los Angeles 24, California

ASSOCIATE EDITORS
E. F. BECKENBACH
D. DERRY
H. L. ROYDEN
E. G. STRAUS
T. M. CHERRY
M. OHTSUKA
E. SPANIER
F. WOLF

\section{SUPPORTING INSTITUTIONS}

UNIVERSITY OF BRITISH COLUMBIA

CALIFORNIA INSTITUTE OF TECHNOLOGY

UNIVERSITY OF CALIFORNIA

MONTANA STATE UNIVERSITY

UNIVERSITY OF NEVADA

NEW MEXICO STATE UNIVERSITY

OREGON STATE COLLEGE

UNIVERSITY OF OREGON

OSAKA UNIVERSITY

UNIVERSITY OF SOUTHERN CALIFORNIA
STANFORD UNIVERSITY

UNIVERSITY OF TOKYO

UNIVERSITY OF UTAH

WASHINGTON STATE COLLEGE,

UNIVERSITY OF WASHINGTON

AMERICAN MATHEMATICAL SOCIETY

CALIFORNIA RESEARCH CORPORATION

HUGHES AIRCRAFT COMPANY

SPACE TECHNOLOGY LABORATORIES

NAVAL ORDNANCE TEST STATION

Printed in Japan by International Academic Printing Co., Ltd., Tokyo, Japan

Reprinted 1966 in the United States of America 


\section{Pacific Journal of Mathematics}

\section{Vol. 11, No. $1 \quad$ November, 1961}

A. A. Albert, Generalized twisted fields ............................ 1

Richard Arens, Operational calculus of linear relations ................... 9

John Herbert Barrett, Disconjugacy of a self-adjoint differential equation of the fourth order ....................................... 25

Paul Richard Beesack, Hardy's inequality and its extensions ............... 39

Julius Rubin Blum and David Lee Hanson, On invariant probability measures.

II .............................................

Robert Allen Bonic, Symmetry in group algebras of discrete groups.......... 73

R. Creighton Buck, Multiplication operators ...................... 95

Jack Gary Ceder, Some generalizations of metric spaces ................. 105

Meyer Dwass, Random crossings of cumulative distribution functions ......... 127

Albert Edrei, Wolfgang H. J. Fuchs and Simon Hellerstein, Radial distribution and

deficiencies of the values of a meromorphic function ............... 135

William Cassidy Fox, Harmonic functions with arbitrary local singularities ..... 153

Theodore Thomas Frankel, Manifolds with positive curvature ............... 165

Avner Friedman, A strong maximum principle for weakly subparabolic

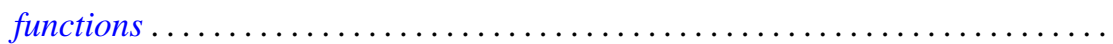

Watson Bryan Fulks and J. O. Sather, Asymptotics. II. Laplace's method for

multiple integrals ......................................

Adriano Mario Garsia and Eugene Richard Rodemich, An embedding of Riemann

surfaces of genus one ..................................... 193

Irving Leonard Glicksberg, Weak compactness and separate continuity......... 205

Branko Grünbaum, On a conjecture of H. Hadwiger .................. 215

Frank J. Hahn, On the action of a locally compact group on $E_{n} \ldots \ldots \ldots \ldots \ldots . . \ldots 221$

Magnus R. Hestenes, Relative hermitian matrices ..................... 225

G. K. Kalisch, On similarity invariants of certain operators in $L_{p} \ldots \ldots \ldots \ldots .247$

Yitzhak Katznelson and Walter Rudin, The Stone-Weierstrass property in Banach

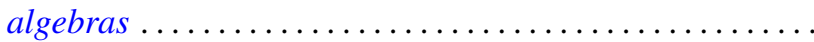

Samir A. Khabbaz, The subgroups of a divisible group $G$ which can be represented as intersections of divisible subgroups of $G \ldots \ldots \ldots \ldots \ldots \ldots \ldots \ldots \ldots . \ldots \ldots 7$

Marvin Isadore Knopp, Construction of a class of modular functions and forms .......................................... 275

Charles Alan McCarthy, Commuting Boolean algebras of projections .......... 295

T. M. MacRobert, Transformations of series of E-functions ................ 309

Heinz Renggli, An inequality for logarithmic capacities ................. 313

M. S. Robertson, Applications of the subordination principle to univalent functions .......................................... 315

David Sachs, Partition and modulated lattices ..................... 325

Frank S. Scalora, Abstract martingale convergence theorems ............... 347

Elbert A. Walker, Torsion endomorphic images of mixed Abelian groups ........ 375

Morgan Ward, The prime divisors of Fibonacci numbers................. 379

Charles R. B. Wright, On the nilpotency class of a group of exponent four....... 387 